\title{
Agresividad y conducta antisocial en individuos con dependencia al teléfono móvil: un posible factor criminogénico
}

Isaac Jahir Olmedo Hernández ${ }^{1, a}$, Edmundo Denis Rodríguez* 1, b, María Esther Barradas Alarcón 2, c, Josué Elí Villegas

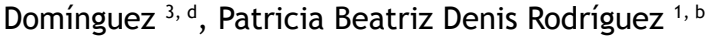

\section{RESUMEN}

Objetivo: Determinar la frecuencia de agresividad y conducta antisocial en estudiantes universitarios con dependencia al teléfono móvil.

Materiales y métodos: Se incluyeron 66 estudiantes universitarios entre 18 a 25 años (54,5 \% eran mujeres). Los participantes completaron la escala Mobile Phone Problematic Use Scale para la dependencia del teléfono móvil y la escala del Inventario Multifásico de la Personalidad de Minnesota, versión 2-RF para comportamiento antisocial y la agresividad.

Resultados: Se encontró dependencia del teléfono móvil en el 22,7 \% de la muestra (punto de corte: percentil 80). Además, se observó una significación estadística $(p<0.01)$ en 8 escalas del MPPI-2-RF, que incluían CR4, AG y AGGR-r, relacionadas con la agresión y el comportamiento antisocial.

Conclusiones: Existe una relación entre la dependencia del teléfono móvil y las escalas de agresividad y comportamiento antisocial. Postulamos que la dependencia del teléfono móvil podría ser un factor criminógeno.

Palabras clave: Dependencia; Trastorno de personalidad antisocial; Agresión (Fuente DeCS: BIREME).

\section{Aggression and antisocial behavior in individuals with mobile phone dependence: a possible factor conducive to crime}

\section{ABSTRACT}

Objective: To determine the frequency of aggression and antisocial behavior in university students with mobile phone dependence.

Materials and methods: Sixty-six (66) university students from 18 to 25 years old (54.5\% females) participated in the study. They completed the Mobile Phone Problematic Use Scale (MPPUS) to assess the mobile phone dependence, and the Minnesota Multiphasic Personality Inventory 2-RF (MMPI-2-RF) to assess the antisocial behavior and aggression.

Results: Twenty-two point seven percent $(22.7 \%)$ of the sample showed mobile phone dependence (cutoff point: 80th percentile). Additionally, a statistical significance $(p<0.01)$ was observed in eight scales of the MPPI-2-RF, including CR4, AG and AGGR-r, which are related to aggression and antisocial behavior.

Conclusions: There is a relationship between mobile phone dependence and the scales assessing aggression and antisocial behavior. We postulate that mobile phone dependence could be a factor conducive to crime.

Keywords: Dependency; Antisocial personality disorder; Aggression (Source: MeSH NLM).

1. Instituto de Medicina Forense, Universidad Veracruzana. Veracruz, México.

2. Facultad de Psicología, Universidad Veracruzana. Veracruz, México.

3. Facultad de Medicina, Universidad Veracruzana. Veracruz, México.

a. Licenciado en Psicología, Máster en Medicina Forense.

b. Médico Cirujano, Máster en Medicina Forense, Doctor en Ciencias Forenses.

c. Licenciada en Psicología, Máster en Investigación Clínica, Doctora en Educación.

d. Médico Cirujano, Máster en Investigación Clínica.

* Autor corresponsal 


\section{INTRODUCCIÓN}

El uso de teléfonos móviles se ha incrementado dramáticamente en la última década; son utilizados por alrededor de 5,8 billones de personas en el mundo. Los primeros estudios relacionados con el uso del teléfono móvil se centraron, principalmente, en sus ventajas en la vida social. Se argumentó que los teléfonos celulares eran un medio ideal para facilitar la comunicación, incluso, a pesar de la distancia. En los últimos 20 años, se ha enfatizado la eficiencia de los teléfonos celulares para el monitoreo de programas de salud preventiva, como el abandono del hábito de fumar o el autocontrol de la diabetes ${ }^{(1,2)}$.

Es evidente que los teléfonos celulares son un gran atractivo para la población, especialmente para los adolescentes, por las siguientes razones: 1) promueven la autonomía personal, 2) proporcionan prestigio en comparación con sus pares, 3 ) proporcionan innovaciones tecnológicas especialmente atractivas, 4) son una fuente de diversión y entretenimiento y 5) favorecen el establecimiento y mantenimiento de relaciones interpersonales ${ }^{(3)}$.

Un área de preocupación reciente es el uso excesivo o incontrolable de teléfonos celulares y su efecto en las actividades diarias, las finanzas personales y la calidad del sueño, entre otros ${ }^{(4)}$. El uso excesivo de teléfonos celulares puede considerarse una adicción conductual, similar a otras adicciones no químicas como las apuestas excesivas, las compras compulsivas y la adicción a los videojuegos ${ }^{(5)}$. Actualmente, la dependencia del teléfono móvil está incluida en la categoría de "dependencia tecnológica" en el Manual diagnóstico y estadístico de trastornos mentales (DSM). Este cuadro se puede manifestar con el uso excesivo del teléfono móvil, que se evidencia en alto costos de mantenimiento y elevado número de llamadas y mensajes; se presentan problemas con los padres debido al uso excesivo del teléfono; la interferencia con otras actividades escolares 0 personales; un uso cada vez mayor para obtener la misma satisfacción (tolerancia tecnológica), y finalmente, la necesidad de usar la celda constantemente y sentir angustia sin un teléfono. Se debe señalar que hasta ahora no hay límites científicos claros entre los conceptos de uso excesivo, uso inapropiado, dependencia y adicción a los teléfonos celulares ${ }^{(6,7)}$.

En los últimos años se ha utilizado el término "nomofobia" para referirse a la dependencia del teléfono móvil. La palabra "nomofobia" proviene de "fobia a los teléfonos no móviles", que puede traducirse como la fobia de no tener un teléfono móvil cerca ${ }^{(8-10)}$. Aunque se considera un problema de origen multifactorial, algunas pruebas apuntan a un fondo genético, ya que este factor ha sido implicado en ciertas patologías asociadas con el consumo, como las compras compulsivas, la adicción a los videojuegos y otros factores ${ }^{(11,12)}$.

La nomofobia es un fenómeno de frecuencia creciente en casi todo el mundo ${ }^{(8)}$. En un estudio realizado en 415 estudiantes indios, la frecuencia de nomofobia fue de $31,33 \%$ y se encontró una fuerte asociación con el género, el tiempo promedio de uso diario y la edad de uso ${ }^{(13)}$. Por otro lado, en un grupo de 300 estudiantes suecos, la frecuencia de nomofobia fue del $17 \%$ (14). En España, la frecuencia de la dependencia tecnológica se analizó en 5538 estudiantes de 12 a 20 años, y se encontró que la dependencia de internet era del 13,6 \%; la dependencia de los teléfonos celulares era $2,4 \%$, y la dependencia de los videojuegos, 6,2\% (15). Finalmente, en una muestra de 1519 estudiantes suizos, la frecuencia de nomofobia fue del $16,9 \%{ }^{(16)}$.

Medir el grado de dependencia del teléfono móvil no es una tarea fácil (17). Existen numerosos estudios en los que los parámetros o escalas utilizadas son muy diversos, lo que impide un análisis comparativo. Una de las escalas más utilizadas es la escala de uso problemático para teléfonos móviles (MPPUS), que se basa en 27 parámetros que miden la tolerancia, el rango de abstinencia, el uso prolongado del dispositivo, la reducción o suspensión de actividades debidas al uso del teléfono, la existencia de problemas físicos o psicológicos derivados de la dependencia y la persistencia del uso a pesar de la aparición de la familia o problemas personales. Cada parámetro se basa en una escala de Likert de 5 niveles, donde 1 corresponde a un desacuerdo total y 5 corresponde a un acuerdo total, con puntos intermedios (18). Esta escala incluye preguntas escritas en inglés, por lo que es necesario validarla en la población de habla hispana para determinar su aplicabilidad ${ }^{(19)}$. Su uso fue validado recientemente en una población española, en la que se encontró un valor alfa de Cronbach de $0.928{ }^{(20)}$. Recientemente, esta escala se validó en una población mesoamericana, que incluye México ${ }^{(21)}$.

La frecuencia de los problemas mentales está aumentando en todo el mundo. El uso creciente de teléfonos celulares se ha estudiado recientemente como un factor que podría alterar la génesis y la epidemiología de los trastornos mentales, especialmente en relación con el efecto de la radiación electromagnética en la fisiología cerebral ${ }^{(22,23)}$. Varias investigaciones han tratado de vincular el uso excesivo de teléfonos celulares con la aparición de síntomas y síndromes psicosociales ${ }^{(12)}$. En general, la dependencia del teléfono móvil se ha relacionado con ansiedad, 
trastornos del sueño y trastornos depresivos (24,25). Recientemente, en una muestra de 127 adolescentes, se encontró una conexión importante entre los trastornos de ansiedad y la dependencia del teléfono móvil, especialmente en relación con el número de llamadas y de mensajes recibidos (26); y se reportan hallazgos similares en un grupo de 263 estudiantes europeos (27). En un estudio de población que incluyó 1455 hombres y 2701 mujeres, todos ellos en edad universitaria, la prevalencia de los síntomas de ansiedad fue del $29 \%$ en las personas con dependencia de teléfonos móviles (28). Una investigación reciente de 380 estudiantes asiáticos con nomofobia, reporta una frecuencia de trastornos del sueño del $61,7 \%$. La dependencia del teléfono móvil se ha relacionado con trastornos depresivos, ya sea como factor predictivo o como asociado ${ }^{(29,30)}$, y también con la ideación suicida y el intento de suicidio ${ }^{(31)}$.

Además de las características psicopatológicas mencionadas anteriormente, la dependencia de los teléfonos móviles se conecta con la aparición de comportamientos antisociales y agresivos que, en ciertas circunstancias, podrían hacer de la nomofobia un factor de riesgo criminológico ${ }^{(32-34)}$. El primer informe conocido fue el de un individuo con agorafobia, agresividad y nomofobia de más de 15 años de evolución, el tratamiento farmacológico y conductual permitió eliminar las fobias, con la excepción de la dependencia del teléfono móvil (35). Diversos estudios relacionan la agresión y la nomofobia, aunque la dificultad radica en los criterios para definir el comportamiento impulsivo, agresivo y antisocial. En un estudio realizado en una muestra de 11110 estudiantes de Taiwán, se observó que el comportamiento agresivo era más frecuente en aquellos que tenían dependencia del teléfono móvil (OR 2,30, $\mathrm{p}<0.01)^{(36)}$. En un estudio similar realizado con 18104 adolescentes asiáticos, del grupo que padecía nomofobia, el $12,1 \%$ había participado en conductas de acoso agresivo (37). Se obtuvieron resultados similares en un estudio realizado en 14221 estudiantes chinos ${ }^{(38)}$ y 9405 adolescentes japoneses ${ }^{(39)}$, donde el comportamiento agresivo se relacionó con la dependencia de los teléfonos móviles, aunque el diseño de los estudios impide el análisis estadístico.

En el presente estudio, deseamos establecer la relación entre la presencia de dependencia de teléfonos móviles y el comportamiento agresivo, antisocial, o ambos, en estudiantes universitarios.

\section{MATERIALES Y MÉTODOS}

Diseño y población

En el presente estudio, incluimos un grupo de individuos de 18 a 25 años de edad, estudiantes de la carrera de
Histotecnología y Embalsamamiento en la Universidad Veracruzana, de cualquier sexo y lugar de origen y con el antecedente de poseer un teléfono móvil para uso personal por al menos 1 año antes del inicio del estudio. Excluimos a los que tenían antecedentes de padecer o habían padecido un trastorno neuropsiquiátrico, a los que tomaban algún medicamento neurotrópico o que habían sido víctimas de un acto criminal en los 3 meses anteriores al estudio.

\section{Variables y mediciones}

En primer lugar, se les aplicó la escala MPPUS, desarrollada por Bianchi y Phillips en 2005 y validada con un alfa de Cronbach de 0,94 en varios tipos de población, incluida América Latina. Es una escala tipo Likert de 27 elementos con 5 opciones de respuesta que van desde "Totalmente de acuerdo" a "Totalmente en desacuerdo", en la que se analiza diversos aspectos del uso excesivo del teléfono móvil como tiempo de uso, efecto de la vida diaria, aparición de problemas físicos, problemas familiares, alteraciones en el trabajo, etc. Se establece una puntuación que varía de 27 a 135; cuanto mayor sea la puntuación, mayor será el grado de dependencia de los teléfonos móviles. Después de MPPUS, aplicamos el Inventario de personalidad multifásico de Minnesota (MMPI-2-RF), un instrumento ampliamente utilizado para determinar diferentes rasgos de personalidad del individuo, incluida la agresión y el comportamiento antisocial. Para el análisis se seleccionaron las escalas AP (Alteración del pensamiento), AC / PE (Alteraciones del comportamiento), CR1 (quejas somáticas), CR4 (comportamiento antisocial), CR8 (experiencias aberrantes), CR9 (activación hipomaníaca), QCO (quejas cognitivas), ANS (ansiedad), TEN (tendencia al enojo), AG (agresión), EUF (euforia), PFA (problemas familiares), ESO (evitación social), DES (desapego) y AGGR-r (agresividad, revisada). Estas escalas son las que están más relacionadas con los fenómenos estudiados en este trabajo. Cada escala se estimula con un valor numérico T que refleja el grado de afectación de un individuo en particular.

\section{Análisis estadístico}

Los valores de dependencia del teléfono móvil, las variables sociodemográficas y las estimaciones obtenidas en el instrumento MMPI-2-RF se analizaron mediante la prueba de Kruskal-Wallis con post-hoc test de Dunn y el análisis de correlación de Spearman, utilizando el paquete estadístico IBM SPSS en su versión 24.0.

\section{Consideraciones éticas}

Los individuos participantes en el estudio dieron su consentimiento escrito. Se respetaron las implicaciones éticas derivadas de acuerdos internacionales en la 
materia, contenidas en el Acuerdo de Helsinski.

\section{RESULTADOS}

Se incluyeron 66 estudiantes que cumplieron con los criterios de inclusión, con una edad promedio de
22,3 años y $54,5 \%$ de mujeres. Las características sociodemográficas, el número de horas de uso diario y el tiempo total de uso del teléfono móvil se pueden ver en la tabla 1.

Tabla 1. Comparación de valores sociodemográficos y patrones de uso en relación con las categorías de dependencia de teléfonos móviles

\begin{tabular}{|c|c|c|c|c|c|}
\hline \multicolumn{6}{|c|}{ Patrón de uso del teléfono móvil } \\
\hline & & Ocasional & Habitual & De riesgo & Problemático \\
\hline \multirow[t]{2}{*}{ Sexo } & Masculino & $40 \%$ & $51,2 \%$ & $25 \%$ & $66,7 \%$ \\
\hline & Femenino & $60 \%$ & $48,8 \%$ & $75 \%$ & $33,3 \%$ \\
\hline \multirow{2}{*}{$\begin{array}{l}\text { Años de uso del } \\
\text { teléfono móvil }\end{array}$} & $<1$ año & $50 \%$ & $9,8 \%$ & $0 \%$ & $0 \%$ \\
\hline & 1 a 5 años & $50 \%$ & $46,3 \%$ & $16,7 \%$ & $0 \%$ \\
\hline \multirow{5}{*}{ Horas de uso al día } & $>5$ años & $0 \%$ & $43,9 \%$ & $83,3 \%$ & $100 \%$ \\
\hline & $<1$ hora & $10 \%$ & $0 \%$ & $0 \%$ & $0 \%$ \\
\hline & 1 a 3 horas & $70 \%$ & $29,3 \%$ & $0 \%$ & $0 \%$ \\
\hline & 3 a 6 horas & $20 \%$ & $29,3 \%$ & $16,7 \%$ & $0 \%$ \\
\hline & $>6$ horas & $0 \%$ & $41,5 \%$ & $83,3 \%$ & $100 \%$ \\
\hline
\end{tabular}

Con la referencia de los percentiles 15,80 y 95 de los valores obtenidos en la escala MPPUS para dependencia de teléfonos móviles ${ }^{(14)}$, se establecieron cuatro categorías: usuarios ocasionales (por debajo del percentil 15), usuarios habituales (entre el percentil 15 y 80 ), usuarios en riesgo (entre el percentil 80 y 95 ) y usuarios problemáticos (por encima del percentil 95). Según estos valores, el $15,2 \%$ de la muestra fueron usuarios ocasionales, el 62,1\% fueron usuarios regulares, el $18,2 \%$ fueron usuarios en riesgo y el $4,5 \%$ fueron usuarios problemáticos; estableciendo el percentil 80 como punto de corte, establecemos que el $22,7 \%$ de la muestra presenta dependencia de teléfonos móviles, un valor relativamente similar al reportado en otros estudios.

La tabla 2 muestra una comparación entre los valores obtenidos en las escalas MMPI-2-RF y los valores de nomofobia en la población estudiada, en base a la prueba de Kruskal-Wallis.

Tabla 2. Análisis comparativo mediante la prueba de Kruskal-Wallis con post-hoc test de Dunn

\begin{tabular}{|c|c|c|c|c|c|}
\hline & \multicolumn{5}{|c|}{ Patrón de uso del teléfono móvil } \\
\hline & Ocasional $^{a}$ & Habitual $^{b}$ & De riesgo $^{c}$ & Problemático $^{d}$ & $\mathbf{p}$ \\
\hline Alteración del pensamiento & $50,90 \pm 7,80$ & $54,32 \pm 11,34$ & $63,92 \pm 14,79$ & $67,33 \pm 6,35$ & 0.01 \\
\hline Alteración emocional & $40,50 \pm 9,19 b c d$ & $50,78 \pm 10,34 a$ & $59,42 \pm 11,10 a$ & $66,67 \pm 12,90 a$ & $<0.01$ \\
\hline Quejas somáticas & $51,80 \pm 10,76$ & $56,93 \pm 10,69$ & $61,00 \pm 10,95$ & $59,67 \pm 10,02$ & ns \\
\hline Conducta antisocial & $39,60 \pm 10,45 \mathrm{~cd}$ & $51,54 \pm 13,76$ & $56,08 \pm 13,47 a$ & $66,67 \pm 2,89 a$ & $<0.01$ \\
\hline Experiencias aberrantes & $55,10 \pm 10,60$ & $59,59 \pm 12,11$ & $70,42 \pm 15,45$ & $73,00 \pm 5,29$ & 0.01 \\
\hline Activación hipomaniaca & $46,70 \pm 6,78 \mathrm{~cd}$ & $51,93 \pm 10,39$ & $61,50 \pm 9,09 a$ & $65,67 \pm 12,34 a$ & $<0.01$ \\
\hline Quejas cognitivas & $54,50 \pm 10,79$ & $62,95 \pm 13,44$ & $64,67 \pm 12,84$ & $68,67 \pm 8,74$ & ns \\
\hline
\end{tabular}




\begin{tabular}{|c|c|c|c|c|c|}
\hline & \multicolumn{5}{|c|}{ Patrón de uso del teléfono móvil } \\
\hline & Ocasional $^{\mathrm{a}}$ & Habitual $^{b}$ & De riesgoc & Problemático ${ }^{d}$ & $\mathbf{p}$ \\
\hline Ansiedad & $49,20 \pm 8,50$ & $53,44 \pm 14,53$ & $64,25 \pm 14,57$ & $59,67 \pm 23,86$ & ns \\
\hline Enojo & $48,10 \pm 9,52$ & $56,59 \pm 9,91$ & $59,00 \pm 11,79$ & $59,33 \pm 10,69$ & ns \\
\hline Agresión & $48,10 \pm 11,75$ & $55,51 \pm 12,16$ & $64,25 \pm 12,58$ & $69,00 \pm 14,73$ & $<0.01$ \\
\hline Euforia & $49,30 \pm 6,88 c$ & $57,37 \pm 12,01$ & $66,17 \pm 13,00 a$ & $65,33 \pm 7,51$ & $<0.01$ \\
\hline Problemas familiares & $53,50 \pm 8,73$ & $60,61 \pm 14,81$ & $61,50 \pm 9,98$ & $54,67 \pm 17,04$ & ns \\
\hline Evitación social & $47,90 \pm 9,52$ & $52,68 \pm 16,97$ & $52,25 \pm 13,53$ & $54,67 \pm 20,50$ & ns \\
\hline Desapego & $55,00 \pm 13,17$ & $59,63 \pm 17,31$ & $59,08 \pm 15,69$ & $71,00 \pm 24,25$ & ns \\
\hline Agresividad & $47,70 \pm 7,39 c$ & $47,98 \pm 10,60 c$ & $61,25 \pm 9,23 a b$ & $57,33 \pm 16,92$ & $<0.01$ \\
\hline MPPUS & $43,10 \pm 4,12 \mathrm{bcd}$ & $77,80 \pm 18,60$ acd & $99,83 \pm 2,04 a b$ & $107,67 \pm 1,53 a b$ & $<0.01$ \\
\hline Edad & $20,90 \pm 2,47$ & $21,10 \pm 2,08$ & $20,83 \pm 2,21$ & $22,33 \pm 1,15$ & ns \\
\hline
\end{tabular}

Datos expresados en media \pm desviación estándar.

Nivel de significancia con valor de $p<0,05$.

Diferencias significativas entre grupos se identifican con letras diferentes en misma fila (abcd).

ns = no significativo. $\mathrm{SD}=$ Desviación estándar

En la tabla 3 hacemos un análisis de correlación de Spearman entre las escalas MMPI-2-RF utilizadas en nuestra población, el cual determina la correlación entre la edad y las escalas relacionadas con alteraciones del pensamiento, quejas cognitivas, conducta antisocial, agresión y agresividad. Del mismo modo, la escala de agresión se correlacionó con las escalas de desapego, conducta antisocial y quejas somáticas.

Tabla 3. Correlación de Spearman. Significación estadística con un valor de $p<0.05$ *, valor $<0.01$ **

\begin{tabular}{|c|c|c|c|c|c|c|c|c|c|c|c|c|c|c|c|c|c|}
\hline & AP & $\mathrm{AE}$ & Qs & CAS & EA & $\mathrm{AH}$ & $Q C$ & AN & TE & AG & EU & PF & ES & DES & AGR & MPPUS & Edad \\
\hline AP & 1 & $.530^{* *}$ & $.368^{\star \star}$ & $.583^{* *}$ & $.870^{* *}$ & $.484^{* *}$ & $.514^{* *}$ & $.499^{* \star}$ & .215 & $.560^{\star *}$ & $.478^{\star \star}$ & $.496^{\star \star}$ & .170 & $.320^{* *}$ & $.288^{*}$ & $.348^{* *}$ & .026 \\
\hline$A E$ & $.530^{\star *}$ & 1 & $.394^{* *}$ & $.806^{* *}$ & $693^{* *}$ & $761^{* *}$ & $.412^{\star \star}$ & $.341^{*}$ & $.448^{* *}$ & $.755^{\star *}$ & $.442^{\star *}$ & .357 & .069 & $.272^{*}$ & $.569^{* \star}$ & $.678^{* *}$ & -.062 \\
\hline Qs & $.368^{* *}$ & $.394^{* *}$ & 1 & $.540^{* *}$ & $.360^{* *}$ & .237 & $.477^{* \star}$ & $.453^{\star *}$ & $.424^{* *}$ & $.347^{* *}$ & $.379^{\star \star}$ & $.360^{* *}$ & $.315^{*}$ & .219 & .003 & $.354^{* *}$ & -.060 \\
\hline CAS & $.583^{* *}$ & $.806^{\star \star}$ & $.540^{\star \star}$ & 1 & $.655^{\star}$ & $.495^{* *}$ & $.504^{* \star}$ & $.387^{* \star}$ & $.469^{* \star}$ & $.608^{* \star}$ & $.351^{\star *}$ & $.538^{\star \star}$ & .180 & $.292^{*}$ & .174 & $.646^{* *}$ & -.019 \\
\hline$E A$ & $.870^{\star *}$ & $.693^{* *}$ & $.360^{\star *}$ & $.655^{\star *}$ & 1 & $.648^{* *}$ & $.595^{\star *}$ & $.472^{\star \star}$ & $.253^{*}$ & $.634^{* *}$ & $.521^{\star *}$ & $.513^{\star *}$ & .150 & $.355^{\star *}$ & $.441^{* *}$ & $.468^{* *}$ & .008 \\
\hline $\mathrm{AH}$ & $.484^{* *}$ & $.761^{* *}$ & .237 & $.495^{\star *}$ & $.648^{* *}$ & 1 & $.379^{\star \star}$ & $.378^{\star \star}$ & $.416^{* *}$ & $.654^{* *}$ & $.654^{* *}$ & $.268^{\star}$ & .007 & .074 & $.752^{\star *}$ & $.593^{* *}$ & -.027 \\
\hline QC & $.514^{\star *}$ & $.412^{* *}$ & $.477^{\star \star}$ & $.504^{\star \star}$ & $.595^{\star *}$ & $.379^{* *}$ & 1 & $.488^{\star \star}$ & $.353^{* *}$ & $.433^{* *}$ & $.481^{* *}$ & $.457^{\star \star}$ & .169 & $.419^{* \star}$ & .051 & $.366^{* *}$ & -.027 \\
\hline AN & $.499^{\star \star}$ & $.341^{* *}$ & $.453^{\star \star}$ & $.387^{\star *}$ & $.472^{* *}$ & $.378^{* *}$ & $.488^{* *}$ & 1 & .188 & $.319^{* *}$ & $.502^{\star *}$ & $.348^{* *}$ & .114 & .131 & .170 & $.458^{* *}$ & -.016 \\
\hline TE & .215 & $.448^{* *}$ & $.424^{* *}$ & $.469^{* *}$ & $.253^{*}$ & $.416^{* *}$ & $.353^{\star *}$ & .188 & 1 & $.441^{* *}$ & .218 & $.331^{* \star}$ & $.277^{\star}$ & $.266^{*}$ & .234 & $.489^{* \star}$ & .055 \\
\hline$A G$ & $.560^{\star *}$ & $.755^{\star \star}$ & $.347^{\star \star}$ & $.608^{* *}$ & $.634^{* *}$ & $.654^{* *}$ & $.433^{* *}$ & $.319^{\star \star}$ & $.441^{* \star}$ & 1 & $.307^{*}$ & $.412^{\star \star}$ & $.283^{*}$ & $.361^{* \star}$ & $.545^{\star \star}$ & $.589^{\star \star}$ & -.024 \\
\hline EU & $.478^{\star \star}$ & $.442^{* *}$ & $.379^{\star \star}$ & $.351^{* *}$ & $.521^{* *}$ & $.654^{* *}$ & $.481^{* *}$ & $.502^{\star \star}$ & .218 & $.307^{\star}$ & 1 & .196 & -.004 & -.061 & $.378^{\star *}$ & $.399^{\star *}$ & .146 \\
\hline PF & $.496^{\star \star}$ & $.357^{* *}$ & $.360^{\star *}$ & $.538^{* *}$ & $.513^{* *}$ & $.268^{\star}$ & $.457^{\star *}$ & $.348^{* *}$ & $.331^{* *}$ & $.412^{\star *}$ & .196 & 1 & $.301^{*}$ & $.270^{*}$ & -.079 & $.351^{\star *}$ & -109 \\
\hline ES & .170 & .069 & $.315^{\star}$ & .180 & .150 & .007 & .169 & .114 & $.277^{\star}$ & $.283^{*}$ & -.004 & $.301^{*}$ & 1 & $.566^{* \star}$ & -.089 & .194 & -190 \\
\hline DES & $.320^{\star *}$ & $.272^{*}$ & .219 & $.292^{*}$ & $.355^{* *}$ & .074 & $.419^{* *}$ & .131 & $.266^{*}$ & $.361^{* *}$ & -.061 & $.270^{*}$ & $.566^{* *}$ & 1 & -.040 & .238 & -.184 \\
\hline AGR & $.288^{*}$ & $.569^{* *}$ & .003 & .174 & $.441^{* *}$ & $.752^{* *}$ & .051 & .170 & .234 & $.545^{* *}$ & $.378^{\star *}$ & -.079 & -.089 & -.040 & 1 & $.376^{* *}$ & .009 \\
\hline MPPUS & $.348^{* *}$ & $.678^{\star *}$ & $.354^{* *}$ & $.646^{* *}$ & $.468^{* *}$ & $.593^{* *}$ & $.366^{* *}$ & $.458^{\star \star}$ & $.489^{\star *}$ & $.589^{\star \star}$ & $.399^{\star *}$ & $.351^{* *}$ & .194 & .238 & $.376^{* \star}$ & 1 & .024 \\
\hline Edad & .026 & -.062 & -.060 & -.019 & .008 & -.027 & -.027 & -.016 & .055 & -.024 & .146 & -.109 & -.190 & -.184 & .009 & .024 & 1 \\
\hline
\end{tabular}

AP: Alteración del pensamiento; AE: Alteración emocional; Qs: Quejas cognitivas; CAS: Conducta antisocial; EA: Experiencias aberrantes; AH: Activación hipomaníaca; QC: Quejas cognitivas; AN: Ansiedad; TE: Tendencia al enojo; AG: Agresión; EU: Euforia; PF: Problemas Familiares; ES: Evitación Social; DES: Desapego; AGR: Agresividad. 


\section{DISCUSIÓN}

El uso de los teléfonos móviles es parte del desarrollo tecnológico que la sociedad ha experimentado en los últimos 30 años; constituye un excelente medio para entablar un medio de comunicación más efectivo y en menos tiempo. Esto ha sido especialmente importante en individuos jóvenes, quienes hacen uso frecuente del teléfono móvil como parte de sus actividades cotidianas.

El uso desmedido del teléfono móvil es un fenómeno social que ha ido adquiriendo importancia epidemiológica de forma paulatina; existen reportes en todo el mundo acerca del aumento desmedido del uso del teléfono móvil, especialmente en adolescentes. Para poder determinar la magnitud real de esta problemática, se han realizado diversos estudios epidemiológicos al respecto; en ellos se ha observado que la frecuencia de uso desmedido del teléfono móvil es variable, dependiendo del tipo de escala utilizada y la población analizada. La prevalencia observada oscila entre $5,8 \%$ y $33 \%{ }^{(4)}$. En nuestra población, la frecuencia de uso desmedido de teléfono móvil fue $22,4 \%$, cifra relativamente similar a lo reportado en la literatura latinoamericana y europea ${ }^{(5)}$.

Los motivos por los que un determinado individuo puede desarrollar dependencia al teléfono móvil aún se encuentran en estudio. Se ha sugerido que está relacionado con la edad, el sexo y la ocupación, así como la presencia previa de patologías psicosociales diversas, como los trastornos de ansiedad, sin embargo, los resultados son contradictorios. Dado que nuestra muestra es pequeña, resulta difícil analizar dichas circunstancias, pero encontramos cierta relación entre el grado de dependencia al teléfono móvil y algunas características de tipo demográfico, como el sexo masculino, el uso del teléfono móvil por más de 6 horas al día, así como emplear el teléfono móvil por más 5 años (desde el momento en que se usó por primera vez).

Ha surgido la preocupación relacionada con las implicaciones sociales y sanitarias de la dependencia al teléfono móvil, especialmente en población adolescente (36). En algunos estudios se ha sugerido que pudiera estar relacionada con la presencia de trastornos del sueño (particularmente insomnio), trastornos de ansiedad y patologías de tipo afectivo (especialmente depresión) ${ }^{(37)}$. Aún no se ha estudiado la relación entre dependencia al teléfono móvil y conducta agresiva o antisocial, aunque en dos estudios poblacionales previos se sugirió una posible relación ${ }^{(38,39)}$. Para ello, decidimos implementar el Inventario Multifásico de Personalidad de Minnesota en una población joven, se trata de una escala psicométrica frecuentemente usada en todo el mundo y validada en nuestra población. Además, utilizamos diversas escalas relacionadas directa o indirectamente con la agresividad y la conducta antisocial.

Observamos que, a mayor valor de dependencia al teléfono móvil, se presentaba un valor estadísticamente mayor en las siguientes escalas del Inventario Multifásico: AP (alteración del pensamiento), AC/PE (alteraciones emocionales), CR4 (comportamiento antisocial), CR8 (experiencias aberrantes), CR9 (activación hipomaníaca), AG (agresión), EUF (euforia) y AGGR-r (agresividad revisada). Las escalas antes mencionadas están relacionadas con la impulsividad, la baja tolerancia a los estímulos, la tendencia a reaccionar con la agresión y el desarrollo de conductas antisociales.

Es bien sabido que la agresividad y la conducta antisocial son factores que hacen que un individuo tenga mayor riesgo de involucrarse en actividades de tipo delictivo; por ese motivo, los hallazgos de esta investigación tienen importancia criminológica, especialmente en la posibilidad de que el número cada vez mayor de individuos con dependencia al teléfono móvil pudiera estar vinculado con actividades y conductas de riesgo para al desarrollo de un comportamiento pro-criminal. Podríamos estar observando la punta de un iceberg en relación a un fenómeno social con grandes implicaciones en el mediano plazo.

Nuestro estudio tiene algunos sesgos que limitan su análisis; el primero de ellos se relaciona con el tipo y tamaño de muestra; su análisis solo puede realizarse en población joven y con características demográficas similares. Por otro lado, aunque el instrumento de medición es confiable, debe tomarse en cuenta que existen poblaciones específicas que requieren un abordaje distinto, como la población intracarcelaria o aquella perteneciente a grupos etarios distintos.

En conclusión, nuestro trabajo establece una relación entre la dependencia del teléfono móvil y las escalas de agresividad y comportamiento antisocial por lo que planteamos que la dependencia del teléfono móvil podría ser un factor criminógeno.

Es importante realizar estudios similares en muestras de poblaciones más grandes con diferentes características sociodemográficas, para determinar si los resultados obtenidos se pueden extrapolar a otras regiones geográficas y otros grupos de población; de ser así, será importante diseñar estrategias preventivas que permitan detectar temas de riesgo en los que su dependencia de teléfonos móviles podría involucrarlos en el comportamiento delictivo. 


\section{REFERENCIAS BIBLIOGRÁFICAS}

1. Billieux J. Problematic use of the mobile phone: a literature review and a pathway model. Curr Psychiatry Rev. 2012; 8(4): 299-307.

2. Bianchi A, Phillips JG. Psychological predictors of problem mobile phone use. Cyberpsychol Behav. 2005; 8(1): 39-51.

3. Ruiz-Olivares R, Lucena V, Pino MJ, Herruzo J. Analysis of behavior related to use of the Internet, mobile telephones, compulsive shopping and gambling among university students. Adicciones. 2010; 22(4): 301-9.

4. Desai NR, Kesari KK, Agarwal A. Pathophysiology of cell phone radiation: oxidative stress and carcinogenesis with focus on male reproductive system. Reprod Biol Endocrinol. 2009; 7(1): 114

5. Pedrero Pérez EJ, Rodríguez Monje MT, Ruíz Sánchez De León JM. Mobile phone abuse or addiction: a review of the literature. Adicciones. 2012; 24(2): 139-52.

6. Bragazzi NL, Del Puente G. A proposal for including nomophobia in the new DSM-V. Psychol Res Behav Manag. 2014; 7(1): 155-60.

7. Chóliz M. Mobile phone addiction: a point of issue. Addiction. 2010; 105(2): 373-4.

8. Takao M, Takahashi S, Kitamura M. Addictive personality and problematic mobile phone use. Cyberpsychol Behav. 2009; 12(5): 501-7.

9. Peraman R, Parasuraman S. Mobile phone mania: arising global threat in public health. J Nat Sci Biol Med. 2016; 7(2): 198-200.

10. Spear King AL, Martins Valenca A, Cardoso Silva A, Sancassiani F, Machado S, Egidio Nardi A. "Nomophobia": impact of cell phone use interfering with symptoms and emotions of individuals with panic disorder compared with a control group. Clin Pract Epidemiol Ment Health. 2014; 10(1): 28-35.

11. Miller G, Zhu G, Wright MJ, Hansell NK, Martin NG. The heritability and genetic correlates of mobile phone use: a twin study of consumer behavior. Twin Res Hum Genet. 2012; 15(1): 97-106.

12. Augner C, Hacker GW. Association between problematic mobile phone use and psychological parameters in young adults. Int J Public Health. 2012; 57(2): 437-41.

13. Nikhita CS, Jadhav PR, Ajinkya SA. Prevalence of mobile phone dependence in secondary school adolescents. J Clin Diagn Res. 2015; 9(11): 6-9.

14. Smetaniuk $P$. A preliminary investigation into the prevalence and prediction of problematic cell phone use. J Behav Addict. 2014; 3(1): 41-53.

15. Muñoz-Miralles R, Ortega-González R, López-Morón MR, Batalla-Martínez C, Manresa JM, Montellá-Jordana N, et al. The problematic use of information and communication technologies (ICT) in adolescents by the cross sectional JOITIC study. BMC Pediatr. 2016; 16(1): 140-7.

16. Haug S, Castro RP, Kwon M, Filler A, Kowatsch T, Schaub MP. Smartphone use and smartphone addiction among young people in Switzerland. J Behav Addict. 2015; 4(4): 299-307.

17. Roberts JA, Petnji Yaya LH, Manolis C. The invisible addiction: cell-phone activities and addiction among male and female college students. J Behav Addict. 2014; 3(4): 254-65.

18. Chóliz M, Pinto L, Phansalkar SS, Corr E, Mujjahid A, Flores C, et al. Development of a brief multicultural version of the test of Mobile Phone Dependence (TMDbrief) Questionnaire. Front
Psychol. 2016; 7(71): 650-9.

19. López-Fernández $O$, Honrubia-Serrano L, Freixa-Blanxart M. Adaptación española del "Mobile Phone Problem Use Scale" para población adolescente. Adicciones. 2012; 24(2): 123-30.

20. Gutiérrez-Puertas L, Márquez-Hernández VV, AguileraManrique G. Adaptation and validation of the spanish version of the Nomophobia Questionnaire in nursing students. Comput Inform Nurs. 2016; 34(10): 470-5.

21. Merlo LJ, Stone AM, Bibbey A. Measuring problematic mobile phone use: development and preliminary psychometric properties of the PUMP scale. J Addiction. 2013; 2013(4): 1-7.

22. Khan MM. Adverse effects of excessive mobile phone use. Int J Occup Med Environ Health. 2008; 21(4): 289-93.

23. Imamura A, Nishida A, Nakazawa N, Shimodera S, Tanaka G, Kinoshita $\mathrm{H}$, et al. Effects of cellular phone email use on the mental health of junior high school students in Japan. Psychiatry Clin Neurosci. 2009; 63(5): 701-3.

24. Mohammadbeigi A, Absari R, Valizadeh $F$, Saadati $M$, Sharifimoghadam S, Ahmadi A, et al. Sleep quality in medical students: the impact of over-use of mobile cell-phone and social networks. J Res Health Sci. 2016; 16(1): 46-50.

25. Ghasempour A, Mahmoodi-Aghdam M. The role of depression and attachment styles in predicting student's addiction to cell phones. Addict Health. 2015; 7(3-4): 192-7.

26. Gao Y, Li A, Zhu T, Liu X, Liu X. How smartphone usage correlates with social anxiety and loneliness. Peer J. 2016; 4(3): e2197.

27. Körmendi A. Smartphone usage among adolescents. Psychiatr Hung. 2015; 30(3): 297-302.

28. Thomée S, Härenstam A, Hagberg $M$. Mobile phone use and stress, sleep disturbances, and symptoms of depression among young adults: a prospective cohort study. BMC Public Health. 2011; 11(1): 66-72.

29. Demirci K, Akgonul M, Akpinar A. Relationship of smartphone use severity with sleep quality, depression and anxiety in university students. J Behav Addict. 2015; 4(2): 85-92.

30. Sansone RA, Sansone LA. Cell phones: the psychosocial risks. Innov Clin Neurosci. 2013; 10(1): 33-7.

31. Oshima N, Nishida A, Shimodera S, Tochigi M, Ando S, Yamasaki $\mathrm{S}$, et al. The suicidal feelings, self-injury and mobile phose use after lights out in adolescents. J Pediatr Psychol. 2012; 37(9): 1023-30.

32. Jacobson C, Bailin A, Milanaik R, Adesman A. Adolescent health implications of new age technology. Pediatr Clin North Am. 2016; 63(1): 183-94.

33. Ehrenreich SE, Beron KJ, Underwood MK. Social and physical aggression trajectories from childhood through late adolescence: predictors of psychosocial maladjustment at age 18. Dev Psychol. 2016; 52(3): 457-62.

34. McConville DW, Cornell DG. Aggressive attitudes predict aggressive behavior in middle school students. J Emot Behav Disord. 2013; 11(3): 179-87.

35. King AL, Valenca AM, Nardi AE. Nomophobia: the mobile phone in panic disorder with agoraphobia: reducing phobias of worsening of dependence?. Cogn Behav Neurol. 2010; 23(1): 52-4.

36. Yang YS, Yen JY, Ko $\mathrm{CH}$, Cheng CP, Yen CF. The association between problematic cellular phone use and risky behaviors and low self-esteem among Taiwanese adolescents. BMC Public Health. 2010; 10(1): 217.

37. Tochigi M, Nishida A, Shimodera S, Oshima N, Inoue K, Okazaki 
Y, et al. Irregular bedtime and nocturnal cellular phose usage as risk factors for being involved in bullying: a cross-sectional survey of japanese adolescents. PLoS One. 2012; 7(9): e45736.

38. Tao S, Wu X, Wan Y, Zhang S, Hao J, Tao F. Interactions of problematic mobile phone use and psychopathological symptoms with unintentional injuries: a school-based sample of chinese adolescents. BMC Public Health. 2016; 16(1): 88.

39. Ko CH, Yen JY, Liu SC, Huang CF, Yen CF. The associations between aggressive behaviors and internet addiction and online activities in adolescents. J Adolesc Health. 2009; 44(6): 598-605.

Fuentes de financiamiento:

Este artículo ha sido financiado por el Instituto de Medicina Forense de la Universidad Veracruzana.

\section{Conflicto de interés:}

Los autores declaran no tener ningún conflicto de interés.

\section{Correspondencia:}

Edmundo Denis Rodríguez

Dirección: Reyes Heroless/nesquina con Juan Pablo Il, Fraccionamiento Costa Verde, Boca del Río, Veracruz, México. CP 94294.

Teléfono: +52 229 9218741- +52 2292083091

Correo electrónico: eddenis@uv.mx

\section{Recibido: 26 de noviembre de 2018 \\ Evaluado: 10 de febrero de 2019 \\ Aprobado: 16 de mayo de 2019}

( ) La revista. Publicado por Universidad de San Martín de Porres, Perú. (cc) BY Licencia de Creative Commons Artículo en acceso abierto bajo términos de Licencia Creative Commons Atribución 4.0 Internacional. (http://creativecommons.org/licenses/by/4.0/)

\section{ORCID iDs}

Isaac Jair Olmedo Hernández Edmundo Denis Rodríguez María Esther Barradas Alarcón Josué Elí Villegas Domínguez Patricia Beatriz Denis Rodríguez 